\title{
SOME FEATURES OF THE GRINDING PROCESS IN THE PRODUCTION OF POWDER MATERIALS
}

\author{
Yunusov Bakhtiar ${ }^{1}$, Normuminov Jakhongir ${ }^{1}$ \\ ${ }^{1}$ The Tashkent state technical university, after I. Karimov, Tashkent city, Uzbekistan
}

\begin{abstract}
Annotation. The article sets out some of the features of the grinding process in the production of powdery materials. Analysis of the literature review on the current state of the issue under study. The general characteristics of the production of powdery materials are studied, the properties of powdery materials as an object of technological processing, including hydromechanical characteristics, are considered.
\end{abstract}

\section{Introduction}

Grinding of materials in the fuel and energy, mining and metallurgical, chemical and other industries is of great importance. In most cases, grinding increases the phase contact surface of the acting masses (in a heterogeneous environment), since materials such as pigments and fillers are used only in a finely ground state. The nature of the feedstock and the required degree of grinding determine the number of grinding stages and the type of grinding equipment. The process is carried out with classifiers of various types (screen, separator, hydraulic classifier), with the help of which the crushed coarse particles that do not meet the requirements for the final product are returned to the grinder for further processing.

Where solid mineral raw materials are processed or the process must take place in a heterogeneous environment, as well as in the case when a commercial product is supplied to the consumer in the form of powders or powder, grinding of solid material is mandatory.

For drying pasty materials, suspensions and solutions in the chemical industry, an aerofoil drying unit with a layer of inert bodies is used. The drying process in this case is accompanied by the simultaneous grinding of the dried product and its removal by air flow from the dryer. The average residence time of particles in the layer is determined by the rate of evaporation of moisture from the surface and the kinetics of abrasion of the dried material; there is no heating of the material, the residence time is calculated in minutes, so it can be used for drying heatsensitive products. Collisions of inert bodies lead to continuous destruction (surface renewal) of the formed dry film of material on the surface of the inert body, and the process takes place mainly in the first period of drying.

Many works $[1,3]$ provide data on drying a number of pasty materials in a gushing layer with inert bodies - glass beads. In this case, the pastes and suspension were fed from the side of the apparatus into the lower part of the layer in the form of a stream using a pump. A trickle of paste, hitting the zone of high temperatures and velocities, was picked up by the coolant flow, broke into small trickles and covered the surface of glass balls rising with the gas flow. At the same time, there was an intensive drying process and disaggregation.

The idea of using a spouting bed as a crusher for grinding solid material is a logical development of the method of drying suspensions and solutions in a layer of inert solid particles. The technique for carrying out this process requires that the layer contains a certain number of foreign inert particles serving as a mixing medium. Inert particles must be harder and heavier than the particles to be treated and capable of gushing, i.e. should be relatively large, of the same size and allow free flow. These restrictions, however, do not apply to the processed particles, since if their proportion in the layer is small enough, then they do not adversely affect the flow of the layer as a whole.

The theoretical foundations of the grinding process are formulated as follows: if the stress in the material exceeds the internal forces of adhesion of the particles, then it breaks up into smaller parts. If the size of these particles is large, then they are again subjected to destruction until the desired product size is obtained. Determining the amount of energy expended in this case is one of the main problems of the theory of grinding. The first attempt to solve it was made by Rittinger, who suggested that the work expended on grinding is proportional to the newly exposed surface in the ground material. Then, the total work expended on the refinement of the body under consideration is obviously equal to

$$
A=A_{y} F=6 A_{y} D^{2}(i-1) .
$$

Where: Ay - specific work; F - is the surface of the particles; $D^{2}$ - edges of a cubic body; $i$ is the degree of grinding.

The assumption about the direct proportionality of the work of grinding the newly formed surface is valid only when grinding the body by cutting or sawing, when the volume of the processed material practically does not affect the energy consumption. If crushing is carried out by crushing, splitting, impacting, or in any combined way, then it is unfair, since this does not take into account the energy spent on deformation of the body without destruction, and it is shown below that the specific work depends not only on the nature of the materials, but also on the class, degree and method of grinding.

In [2], they gave a solution somewhat different from the considered problem of the theory of grinding. They assumed that the energy required for similar changes in the shape of geometrically similar bodies of the same 
technological composition changes in proportion to the volumes or weights of these bodies. According to the theory of elasticity, the work of elastic deformations of bodies of the same technological composition will be:

$$
\begin{aligned}
& A_{1}=\frac{\sigma^{2} v_{1}}{2 E}=\frac{\sigma^{2} G_{1}}{2 E \gamma} ; \\
& A_{2}=\frac{\sigma^{2} v_{2}}{2 E}=\frac{\sigma^{2} G_{2}}{2 E \gamma} .
\end{aligned}
$$

their attitude:

$$
\frac{A_{1}}{A_{2}}=\frac{v_{1}}{v_{2}}=\frac{G_{1}}{G_{2}},
$$

those. the energy consumption for grinding a given material, other things being equal, is directly proportional to its object or weight. But even this theory is insufficient for solving practical problems. Steadler, attempting to develop this theory, gave it a mathematical form that is acceptable for engineering solutions [5]:

$$
A=3 k c r^{n} D^{3} \text {. }
$$

Since and are experimental constants, and it is known for a given material, the work of grinding the material is a complex function of the number of grinding methods.

Formula (5) has not received practical application, since the expression of the work of grinding through the product of the degree of grinding by the conditional force and the conditional path is not physically justified.

The author of the work [5] came to a similar conclusion, the energy spent on grinding the material is the sum of work going on the deformation of the body and the formation of new surfaces:

$$
A=\frac{\sigma_{P}^{2} v}{2 E}+k r F,
$$

Where: $v$ - is the volume of the deformable body; $k r$ - coefficient of proportionality; $F$ - the newly formed surface during the destruction of the body.

The meaning of expression (6) is that the work of grinding is proportional to both the newly formed surface and the volume of the material being ground. This approach is reflected in later research on grinding.

None of the proposed formulas for determining the work expended on grinding has received widespread use, which is primarily due to the complexity of the problem under consideration: the nature, magnitude and direction of the forces under the influence of which the material is destroyed, as well as the quantitative accounting of the results of destruction, determine its complexity to the highest degree. the desire to describe the grinding process by some generalized equation will hardly solve the main problem of the grinding theory. The above formulas are true only for absolutely elastic materials or materials approaching them. In other cases, the problem should be solved empirically.

Analysis of theoretical and experimental studies of the process of grinding clumping materials in the literature has shown that they have not been sufficiently studied.
According to the performed experiments, real grinding curves $d=d(\tau)$ - have a pronounced S-shaped form (Fig. $1)$.

Physically, this is explained by the fact that at the initial moment the grinding speed is low due to the large particle size of the material, and then it increases and then drops again due to the fact that the particles have decreased in size and are more difficult to grind.

We write the equation of the $\mathrm{S}$ - shaped grinding curve:

$$
\frac{d}{d \tau}(d)=-x_{*}\left(A_{*}-d\right)\left(d-B_{*}\right)
$$

Here: $A^{*}$ and $B^{*}$ are the values of the horizontal asymptotes; $X^{*}$ - characterizes the intensity of grinding.

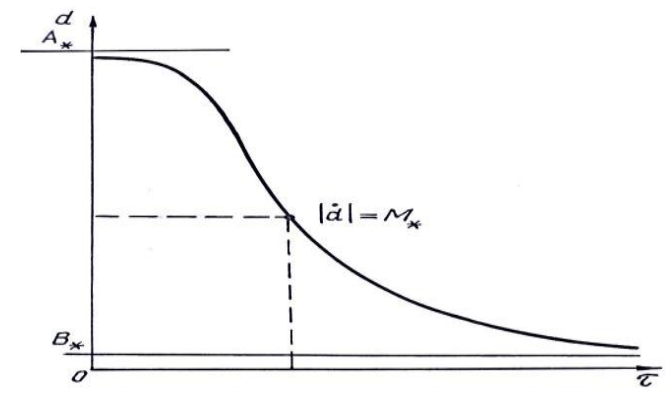

Figure: 1. View of the grinding kinetics curve

$M^{*}$ is the maximum grinding speed at the inflection point of the curve, determined by the external grinding conditions, more precisely, by the kinetic energy of the grinder.

Since, $M^{*}$ - corresponds to the inflection point, then, equating the second derivative $" d$ - to zero, we get:

$$
M_{*}=x_{*} \frac{\left(A_{*}-B_{*}\right)^{2}}{4} \text {. }
$$

From (7) and (8) follows a differential equation that describes the kinetics of grinding:

$$
\frac{d(d)}{d \tau}=-\frac{4 M_{*}}{\left(A_{*}-B_{*}\right)^{2}}\left(A_{*}-d\right)\left(d-B_{*}\right)
$$

Equations (9) describe the kinetics of material particle size reduction.

Thus, the kinetic model of material size change over time in the apparatus is adequate to the experimental values with a $95 \%$ confidence level (Fig. 2.)

Some deviation of the calculated grinding curve from the experimental one can be explained by the complexity of the disaggregation process and the fact that it is difficult to fully take into account the entire set in the model; the assessment of the adequacy of the model according to Fisher's criterion showed that this model satisfactorily describes the process. 


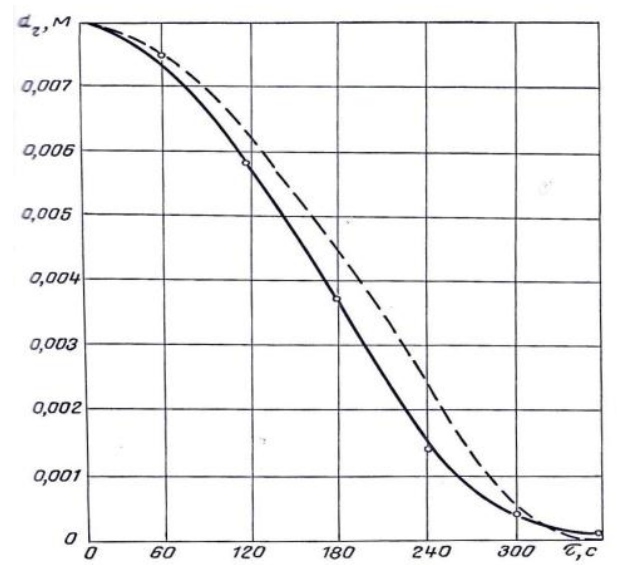

Figure: 2. Curves of grinding cotton powder cellulose factors influencing the kinetics of grinding (CPC). - experimental, - theoretical.

Nevertheless, thus, the analysis of the process showed [4] that:

1) The study of the properties of the material as an object of technological processing and taking into account the mechanical properties makes it possible to select the type of apparatus;

2) New experimental data were obtained on the kinetics of grinding;

\section{Literature}

1) Golubev L.G., Sazhin B.S., Valashek E.R. Drying in the chemical and pharmaceutical industry. $-\mathrm{M}$.: Medicine 1978, p. 271.

2) Romankov P.G., Rashkovskaya N.V. Drying in suspension. -M .: Chemistry, 1968, p. 357.

3) Sazhin B.S. Basics of drying technique. - M . Chemistry, 1984, p. 319.

4) Mukhitdinov D.N., Yunusov B.Kh. and others. Drying of powdered cellulose in an apparatus with a gushing swirling flow of heat carrier in the presence of an inert material. -Khim.prom., 1984, No. 5, p.307.

5) Sidenko P.M. Grinding in the chemical industry. $-\mathrm{M}$.: Chemistry, 1968, p. 331. 